\title{
Review Article \\ Spatial Distributions, Characteristics, and Applications of Craniofacial Stem Cells
}

\author{
Geru Zhang, ${ }^{1}$ Qiwen Li, ${ }^{1}$ Quan Yuan, ${ }^{1,2}$ and Shiwen Zhang $\mathbb{D}^{1,2}$ \\ ${ }^{1}$ State Key Laboratory of Oral Diseases and National Clinical Research Center for Oral Diseases, West China Hospital of Stomatology, \\ Sichuan University, Chengdu, China \\ ${ }^{2}$ Department of Oral Implantology, West China Hospital of Stomatology, Sichuan University, Chengdu, China
}

Correspondence should be addressed to Shiwen Zhang; sw.zhang2018@scu.edu.cn

Received 20 May 2020; Revised 29 July 2020; Accepted 1 August 2020; Published 29 August 2020

Academic Editor: Sangho Roh

Copyright (c) 2020 Geru Zhang et al. This is an open access article distributed under the Creative Commons Attribution License, which permits unrestricted use, distribution, and reproduction in any medium, provided the original work is properly cited.

Stem cells play an irreplaceable role in the development, homeostasis, and regeneration of the craniofacial bone. Multiple populations of tissue-resident craniofacial skeletal stem cells have been identified in different stem cell niches, including the cranial periosteum, jawbone marrow, temporomandibular joint, cranial sutures, and periodontium. These cells exhibit selfrenewal and multidirectional differentiation abilities. Here, we summarized the properties of craniofacial skeletal stem cells, based on their spatial distribution. Specifically, we focused on the in vivo genetic fate mapping of stem cells, by exploring specific stem cell markers and observing their lineage commitment in both the homeostatic and regenerative states. Finally, we discussed their application in regenerative medicine.

\section{Introduction}

The reconstruction of craniofacial bone defects is more challenging than that of the limb bone, as it requires both functional and esthetic recovery. Traditional therapies to regenerate craniofacial bone, including autologous bone grafts, allografts, and xenografts [1-3], exhibit different limitations and often fail to meet the demands of recovery [4-6]. Stem cell-guided regenerative medicine is an alternative that is currently the most promising approach to solve this problem.

Mesenchymal stem cells (MSCs) are groups of cells residing in different tissues and niches, such as the bone marrow, adipose tissue, teeth, and umbilical cord tissue. MSCs have been extensively used in tissue repair, organ reconstruction, immunomodulation, and even in the treatment of disease [7-11]. In addition, self-cell-constituted implantation results in reduced immunogenicity, and the molecules excreted from MSCs are beneficial for tissue recovery $[12,13]$. The combination of MSCs with bioscaffolds further promoted MSC-based therapy by guiding MSC proliferation and migration [14].

To identify and isolate MSCs easily in vitro, the International Society for Cellular Therapy has proposed three criteria to define MSCs [15]. First, the isolated cells can adhere to plastic plates when cultured in vitro. Second, the cells express the CD73, CD90, and CD105 surface markers but not CD34, CD45, CD14 or CD11b, CD79a or CD19, and HLA-DR. Third, the cells can differentiate into osteocytes, chondrocytes, and adipocytes. In addition to in vitro characterization, the recent application and improvement of the fluorescent reporter mouse system and lineage tracing technique make the in vivo study of stem cells feasible [16]. Importantly, the in vivo study of stem cells can aid in accurately recapitulating the niche-dependent functions and interactions of stem cells.

MSCs from bones, including the bone marrow, periosteum, growth plate, and calvarium, have been the most thoroughly studied. It is now recognized that bone MSCs are highly heterogeneous populations that display variable selfrenewal and differentiation potential. MSCs that commit to skeletal lineages and express selective surface markers (e.g., leptin receptor, PDGFR $\alpha$, nestin, Cxcl12, Hox11, PTHrP, Sca1, Ctsk, Axin2, and Gli1) are now defined as skeletal stem cells (SSCs). Craniofacial SSCs are subgroups of cells residing in the calvarium, maxillary and mandibular bones, and 
tooth-supporting tissue. These cells display the basic characteristics of SSCs and are capable of self-renewal and multilineage differentiation. They can regenerate oral tissues and repair critical defects of craniofacial bones [17-20]. However, craniofacial SSCs are distinct from long bone SSCs, which might result from the different developmental origins and stem cell microenvironments/niches. The craniofacial bone originates from the mesoderm and neural crest, and the bony structure is formed by intramembranous ossification. Long bones, on the other hand, mainly originate from the mesoderm and are formed by endochondral ossification [20, 21]. In addition, the craniofacial bone is a flat bone with limited bone marrow, but the long bone is enriched with the bone marrow. Hematopoiesis-depleted or hematopoiesis-enriched-enriched environments result in totally different stem cell niches. Therefore, craniofacial SSCs are different from SSCs in long bones or other tissues. Interestingly, studies have shown that SSCs/MSCs from craniofacial bones exhibit superior osteogenic properties compared with long bone SSCs/MSCs in craniofacial tissue reconstruction [22-24]. A pioneering study also found that postnatal lineage-restricted craniofacial SSCs reverted to their embryonic plastic state and regained a neural crest cell phenotype in response to mandibular distraction for jaw regeneration [25]. Identifying subpopulations and illustrating the properties of craniofacial SSCs are thus crucial to stem cell-guided regenerative medicine.

In this review, we summarize the cranial and maxillofacial tissues in which stem cells reside as well as the characteristics of these stem cells and advancements in their applications.

\section{Periosteum}

The surface of the bone is covered by the periosteum, which is a $50-150 \mu \mathrm{m}$ two-layer membrane with an abundance of nerves and blood vessels (Figure 1). The outer fibrous layer is adjacent to the surrounding soft fibrous and muscular tissue, while the inner layer is highly vascularized and provides a niche for progenitor cells $[26,27]$. A large number of studies have already shown that precursor cells residing in the craniofacial bone periosteum play an important role in bone regeneration [28]. For a long time, no specific cell marker was available to identify and isolate craniofacial periosteumderived stem cells (PSCs) $[28,29]$. Recently, two specific surface makers $\left(\mathrm{Ctsk}^{+}\right.$and $\mathrm{Mxl}^{+} \alpha \mathrm{SMA}^{+}$) have been identified.

Cathepsin K (CTSK) has long been regarded as a specific marker for osteoclasts. Using Ctsk-mGFP transgenic mice to trace cell lineages combined with single-cell RNA sequencing, Debnath et al. identified $\mathrm{Ctsk}^{+}$periosteum stem cells as both long bone and calvarial periosteal skeletal stem cells (PSCs). $\mathrm{Ctsk}^{+}$PSCs are capable of self-renewal, colony formation, and multilineage differentiation. Interestingly, $\mathrm{Ctsk}^{+}$PSCs are highly plastic, as they can mediate not only intramembranous ossification but also endochondral ossification in response to bone injury [30]. In 2019, Park et al. observed that a group of postnatal long-term $\mathrm{Mxl}^{+} \alpha \mathrm{SMA}^{+}$periosteal stem cells contributed significantly to the injury repair of bone defects. In addition to being capable of self-renewal and clonal multipotency, $\mathrm{Mx}^{+} \alpha \mathrm{SMA}^{+}$PSCs can migrate toward the injury site in response to a CCR5 ligand- (CCL5-) dependent mechanism, as visualized by in vivo real-time imaging of the calvarium [31].

\section{Craniofacial Bone Marrow}

Given that jawbones and teeth in the craniofacial system originate from the cranial neural crest, marrow stem cells in jawbones are considered to have characteristics different from those of long bone MSCs. Studies have been performed to compare the similarities and differences between stem cells in the craniofacial, axial, and appendicular regions. Human MSCs in the jawbone and iliac crest have been the most commonly studied, as these sites are ideal for marrow aspiration. Akintoye et al. cultured jawbone MSCs and iliac crest MSCs from the same individual and found that jawbone MSCs displayed a higher proliferation rate, delayed senescence, and greater differentiation potential. In vivo transplantation results showed that jawbone MSCs formed more bone, whereas iliac crest MSCs formed more compacted bone along with hematopoietic tissue [32]. Using tube formation assays and 3D fibrin vasculogenic tests, Du et al. found that jawbone MSCs showed stronger angiogenic propensities than iliac crest MSCs when they were cocultured with human umbilical vein endothelial cells (HUVECs). Coculture with jawbone MSCs allowed HUVECs to form more tube-like structures in vitro and larger vessels in vivo [33]. The increase in the expression of the basic fibroblast growth factor (bFGF) by jawbone MSCs is the key factor contributing to angiogenesis. However, the chondrogenic and adipogenic potential of jawbone MSCs is weaker than that of iliac crest MSCs [34, 35].

Several populations of SSCs in the long bone marrow were identified, including leptin-receptor-expressing $\left(\mathrm{LepR}^{+}\right) \mathrm{SSC}$, nestin-expressing $\left(\mathrm{Nestin}^{+}\right)$SSCs, Gremlin 1-expressing $\left(\mathrm{Grem}^{+}\right)$SSCs, glioma-associated oncogene 1-expressing $\left(\mathrm{Glil}^{+}\right)$SSCs, and CD45 ${ }^{-} \mathrm{Ter}^{-} 119^{-} \mathrm{Tie}^{-} \mathrm{AlphaV}^{+-}$ Thy ${ }^{-6} \mathrm{C}^{-} \mathrm{CD} 105^{-} \mathrm{CD} 200^{+}$SSCs [36-39]. However, their identity and function in the craniofacial bone remain unclear. We recently identified a quiescent population of tissue-resident LepR $^{+}$SSCs in jawbone marrow that became activated in response to tooth extraction and contributed to intramembranous bone formation [40]. Using LepR-Cre; tdTomato; Col2.3GFP reporter mice, we found that these LepR $^{+}$cells remained quiescent in the physiological state and gradually increased in activity with age. External stimuli such as tooth extraction activated LepR ${ }^{+}$SSCs, which rapidly proliferated and differentiated into Col2.3-expressing osteoblasts, contributing significantly to extraction socket repair. Ablation of LepR ${ }^{+}$SSCs with diphtheria toxin dramatically impaired the bone healing process. A mechanistic study showed that alveolar LepR ${ }^{+}$SSCs are responsive to parathyroid hormone/parathyroid hormone I receptor (PTH/PTH1R) signaling. Knockout of Pth1r in the LepR ${ }^{+}$cell lineage disrupted the bone formation process.

\section{Temporomandibular Joint}

The temporomandibular joint (TMJ) is located between the temporal bone and the mandible. It is one of the most frequently used joints in humans and is the only diarthrosis 


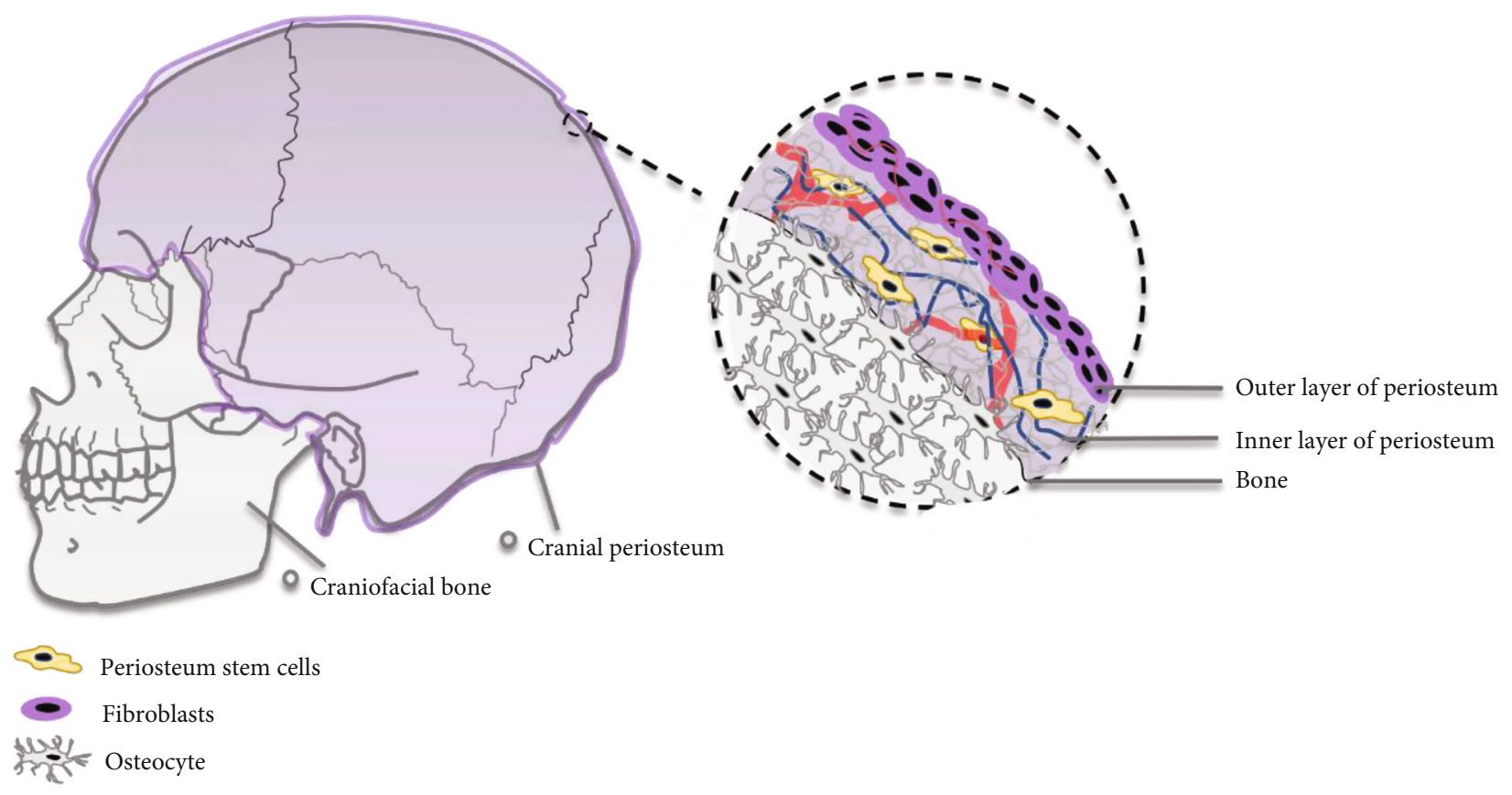

FIGURE 1: Stem cell distribution in the cranial bone. The stem cells are located in the inner layer of calvarium periosteum.

in the stomatognathic system. The occurrence of TMJ osteoarthritis is highly prevalent in humans, yet the regenerative capacity of condylar cartilage is limited. Therefore, identifying and isolating stem cells in the TMJ is crucial for osteoarthritis amelioration and regeneration.

Two types of stem cells reside in the TMJ (Figure 2). One type is TMJ synovium-derived stem cells, and the other type is fibrocartilage stem cells (FCSCs). In 2011, Liu et al. isolated and cultured stem cells from human TMJ synovial fluid and found that these cells exhibit fibroblastic and spindle shapes. Flow cytometry analysis showed that these cells express MSC markers and could be induced to differentiate toward osteogenic, chondrogenic, adipogenic, and neurogenic lineages. Thus, these synovium-derived cells are stem cells [41]. Koyama et al. found STRO-1- and CD146-expressing stem cells in the TMJ synovial fluid of patients with temporomandibular joint disorder. These cells showed great potential to differentiate into chondrocytes, osteoblasts, adipocytes, and neurons [42]. Stem cells were also isolated from the radiolucent zone of TMJ ankylosis patients, but they had a slower proliferation rate and lower osteogenic differentiation capacity than BMSCs [43]. In 2014, Sun et al. isolated synovial fragment cells from the synovial fluid of temporomandibular disease patients and revealed the multilineage differentiation capacity of this group of cells [44]. Fibrocartilage stem cells (FCSCs) reside in the superficial zone of condylar cartilage. A single FCSC could generate a cartilage anlage, which then undergoes autogenous bone formation and supports a hematopoietic microenvironment. Wnt signaling impairs the FCSC niche and results in cartilage degeneration. Intra-articular injection of the Wnt inhibitor sclerostin reconstructed the stem cell niche and repaired TMJ injury, indicating a potential therapeutic strategy for patients with fibrocartilage defects and disease [45].

\section{Sutures}

In summary, the flexible connection between paired calvarial bones permits the deformation of the skull during birth, directs the growth of the skull, and acts as a shock absorber that can cushion the load of mastication (Figure 3). Humans and mice both have four sutures. Metopic sutures (called interfrontal sutures in mice) and sagittal sutures are vertically distributed, and the osteogenic fronts about each other. Coronal sutures and lambdoid sutures are horizontally distributed, and their osteogenic fronts overlap with each other. Unossified sutures are recognized as the bone growth center of the postnatal skull vault [46], where the new bone precipitates at the edges of the bone front. Premature closure of the suture could lead to craniosynostosis. Studies have demonstrated a unique stem cell niche in cranial sutures, where multiple populations ofSeveral subpopulations of suture mesenchymal stem cells (SuSCs) were identified, including Gli1-positive $\left(\mathrm{Gli1}^{+}\right)$cells, Axin2-expressing $\left(\right.$ Axin2 $\left.^{+}\right)$cells, and postnatal Prx1expressing $\left(\operatorname{Prxl}^{+}\right)$cells [46-49]. All these cells possess the ability for self-renewal and continually produce skeletal cell descendants. Clonal expansion analysis demonstrated that SuSCs were capable of forming bones during calvarial development. SuSCs expand dramatically in the damaged site and contribute directly to skeletal repair; the nearer the cells are to the sutures, the better the recovery is $[46,50]$. However, the spatiotemporal properties of SuSCs differ. During the early stage of postnatal development, $\mathrm{Gli1}^{+}$cells were distributed throughout the periosteum, dura, and sutures, whereas Axin $2^{+}$ cells and $\operatorname{Prxl}^{+}$cells appeared only in the sutures. At 4 weeks of age, all of the SuSCs were restricted to the sutures. Gli1 ${ }^{+}$ SuSCs were capable of trilineage differentiation into chondrocytes, osteoblasts, and adipocytes; Axin $2^{+}$SuSCs mainly gave rise to the chondro- and osteolineages, whereas $\operatorname{Prxl}^{+}$SuSCs 


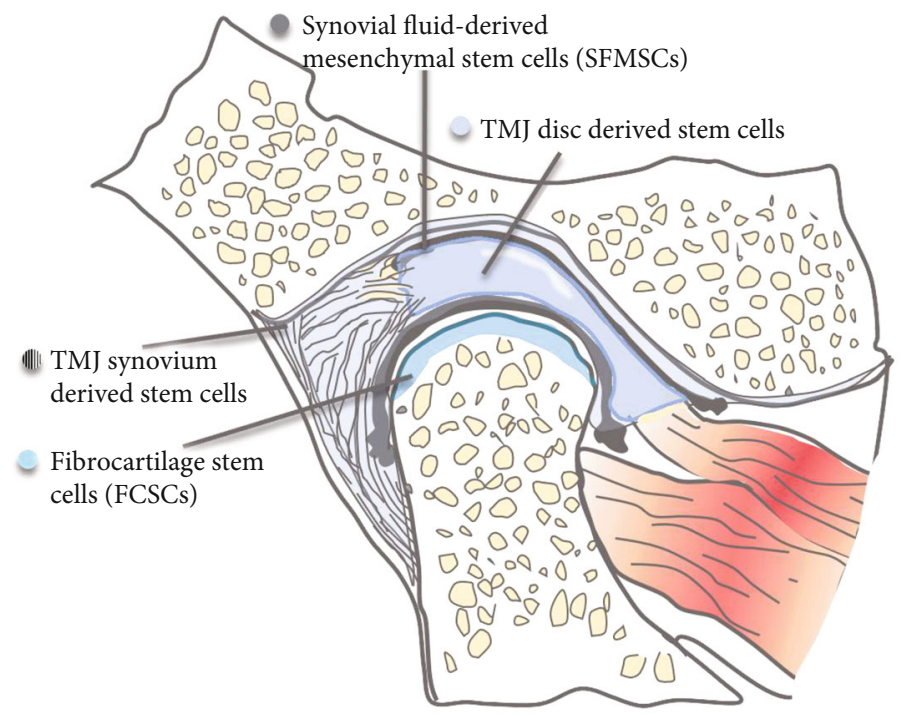

FIGURE 2: Stem cell distribution in TMJ. Stem cells are located in the synovial fluid, synovium, disc of TMJ, and surface zone of condylar cartilage.

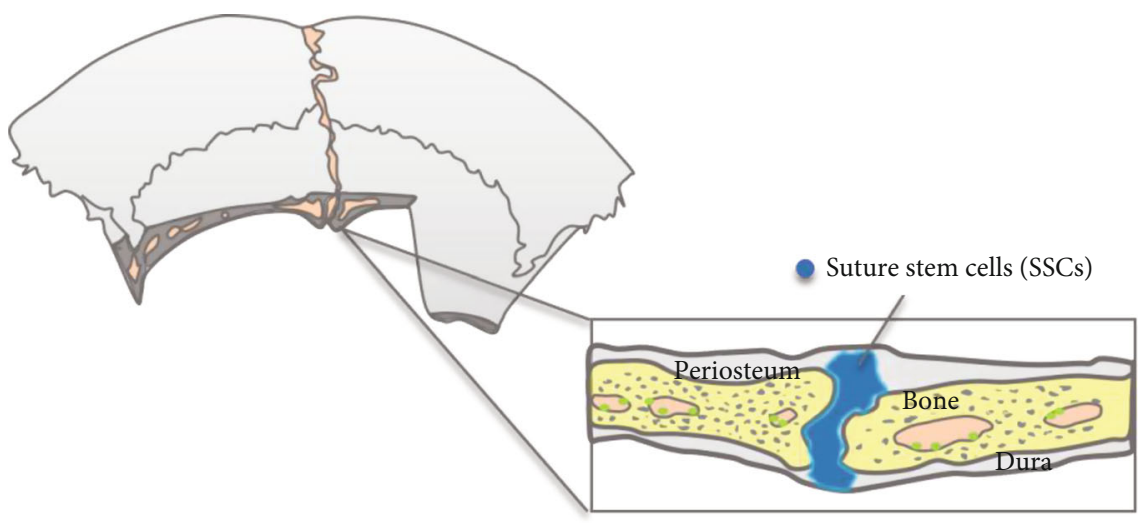

FIgURE 3: Stem cell distribution in sutures.

could only differentiate into osteoblasts. Depletion of $\mathrm{Gli1}^{+}$or Axin $2^{+}$cells but not Prx $1^{+}$SuSCs caused craniosynostosis. Additional comparisons of the three types of cells are listed in Table 1.

\section{Periodontium}

The tooth is a hard tissue containing a vascularized and nerverich pulp chamber and is surrounded by the tooth-supporting periodontium, including the periodontal ligament, cementum, and alveolar bone. Multiple stem cell niches exist in dental tissues, as teeth are uniquely shaped and are subject to complicated microenvironments with occlusal force and microorganisms [53]. To date, more than seven kinds of dental stem cells have been found, including dental pulp stem cells (DPSCs) [54], human exfoliated deciduous teeth (SHED) [55], periodontal ligament stem cells (PDLSCs) [56], dental follicle progenitor cells (DFPCs) [57], stem cells from dental apical papilla (SCAP) [58], tooth germ stem cells (TGSCs) [59], gingival mesenchymal stem cells (GMSCs) [60], and human natal dental pulp stem cells (NDP-SCs) (Figure 4) [61]. All of them are capable of self-renewal, proliferation, and multidi- rectional differentiation [62]. Among them, PDLSCs are the only kind of stem cells that can differentiate into osteoblasts in vivo and contribute to the construction of alveolar bone and tooth extraction sockets.

As reported in 2004, Seo et al. isolated and identified PDLSCs from human impacted wisdom teeth for the first time [56]. In addition to wisdom teeth, PDLSCs can be extracted from permanent tooth root surfaces [63], deciduous tooth [64-66], or even inflammatory periodontal tissues [67]. However, PDLSCs derived from different environments display different properties related to proliferation and osteogenic potential. For example, studies have found that PDLSCs from deciduous teeth promote osteoclastogenesis and lead to root absorption [68]. PDLSCs from inflammatory tissue are predisposed to a pathological local microenvironment [69]. The regulatory mechanism of PDLSC biological behavior remains to be revealed.

To exploit the osteogenic potential of PDLSCs, the osteogenic mechanism of PDLSCs needs to be clarified. It has been reported that antidifferentiation noncoding RNA (ANCR) [70], long noncoding RNAs (lncRNAs) [71], and microRNA182 and microRNA-214 [72, 73] regulate the proliferation 
TABLE 1: The subpopulations of suture mesenchymal stem cells and their characteristics.

\begin{tabular}{|c|c|c|c|c|}
\hline Cell & types & Gli1 $^{+}$cells $[50,51]$ & Axin $2^{+}$cells $[46]$ & Postnatal Prx $1^{+}$cells [52] \\
\hline \multirow{2}{*}{ Distribution } & Early stage & $\begin{array}{l}\text { All over the periosteum, dura, and the } \\
\text { craniofacial sutures }\end{array}$ & In the calvarial sutures & In the calvarial sutures \\
\hline & $\begin{array}{l}\text { One month } \\
\text { after birth }\end{array}$ & Self-renewal & Only in the sutures & l \\
\hline \multirow{3}{*}{ Stemness } & Self-renewal & & Slow-cycling cells & \\
\hline & $\begin{array}{l}\text { Contribution } \\
\text { to other } \\
\text { tissues }\end{array}$ & $\begin{array}{l}\text { Suture mesenchyme, periosteum, dura } \\
\text { mater, and parts of the calvarial bones }\end{array}$ & $\begin{array}{l}\text { Suture mesenchyme and bone } \\
\text { matrix near the osteogenic fronts }\end{array}$ & $\begin{array}{l}\text { All calvarial tissues, except } \\
\text { bone marrow osteoblasts }\end{array}$ \\
\hline & $\begin{array}{l}\text { Ability to } \\
\text { repair the } \\
\text { defect }\end{array}$ & Unequivocal and potentially exclusiv & contribution of the sutural mesench & yyme to calvarial injury repair \\
\hline \multicolumn{2}{|c|}{ Ablation } & Craniosynostosis & Craniosynostosis & $\begin{array}{l}\text { Did not result in } \\
\text { craniosynostosis or any other } \\
\text { major craniofacial phenotype }\end{array}$ \\
\hline \multicolumn{2}{|c|}{ MSC markers } & CD90, CD73, CD44, Sca1, and CD146 & LepR & $\begin{array}{c}\text { Pdgfr } \alpha \text { and Mcam/CD146 } \\
\text { (upregulation), Ccne2, } \\
\text { Mcm4, and Pcna } \\
\text { (downregulation), Itga2, } \\
\text { Itga3, and Itga6 }\end{array}$ \\
\hline \multirow{3}{*}{ Differentiation } & Osteoblasts & + & $\begin{array}{c}+ \\
\text { (upon external stimulation) }\end{array}$ & $\begin{array}{c}+ \\
\text { (stimulated with recombinant } \\
\text { WNT3A) }\end{array}$ \\
\hline & Chondrocytes & + & $\stackrel{+}{\text { (upon external stimulation) }}$ & l \\
\hline & Adipocytes & + & 1 & l \\
\hline \multicolumn{2}{|c|}{ Foundation of each study } & $\begin{array}{l}\text { Glil is the master transcriptional } \\
\text { factor of hedgehog signaling and is } \\
\text { indispensable for bone development } \\
\text { and homeostasis. Gli1+ stem cells } \\
\text { have been identified in canine and } \\
\text { long bones. }\end{array}$ & $\begin{array}{c}\text { Axin2 plays an irreplaceable role in } \\
\text { the Wnt, BMP, and FGF signaling } \\
\text { pathways; Axin2 knockout mice } \\
\text { showed craniosynostosis. }\end{array}$ & $\begin{array}{l}\text { Prx1 was previously shown to } \\
\text { be highly expressed during } \\
\text { limb bud formation and } \\
\text { craniofacial development. }\end{array}$ \\
\hline
\end{tabular}

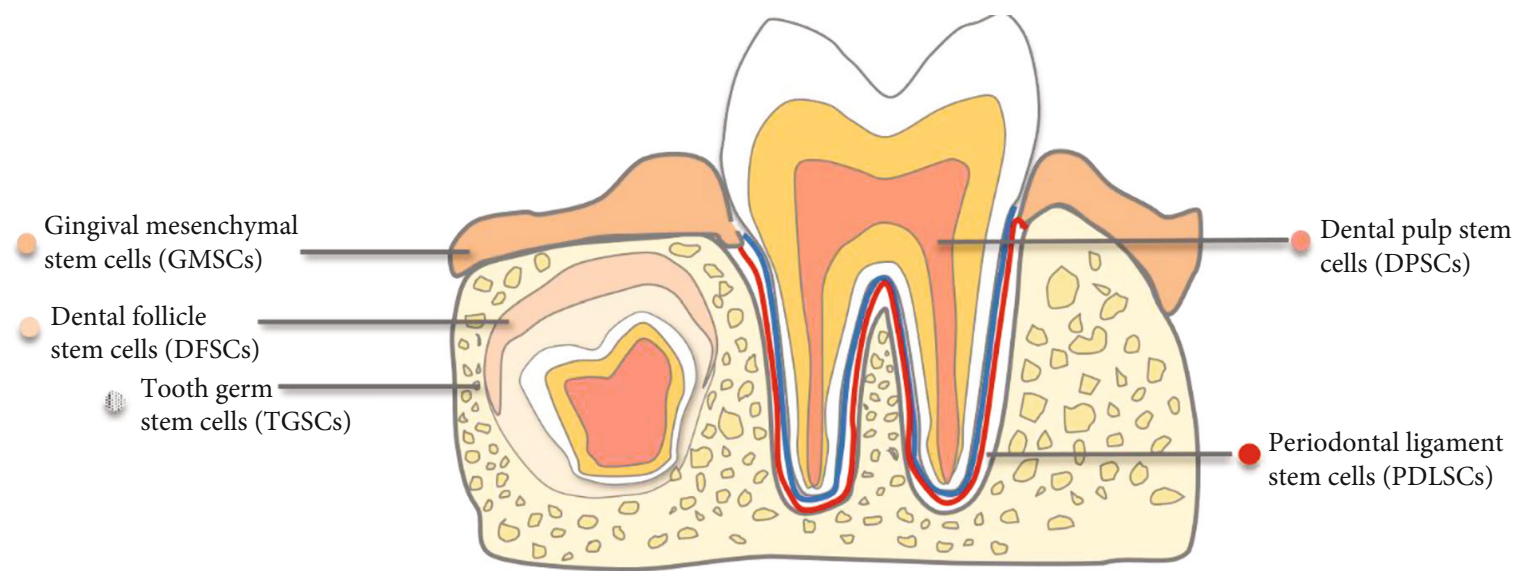

Figure 4: Stem cell distribution in dental tissues. Stem cells are located in gingiva, dental follicle, tooth germ, dental pulp, and periodontal ligament.

and osteogenic differentiation of PDLSCs. He et al. [74] and Yan et al. [75] found that hypoxia and cannabinoid receptor I (CB1) could alter the activity of PDLSCs through the p38/MAPK pathway. Meanwhile, the PI3K-AKT-mTOR pathway [76] and NF- $\kappa$ B axis [77] are also involved in the modula- tion of PDLSCs. In clinical practice, additional topics, such as how metformin contributes to the osteogenic potential of PDLSCs [78] and how nicotine [79, 80] and Porphyromonas gingivalis [81] weaken the osteogenic potential of PDLSCs, remain to be investigated. 
PDLSCs exhibit high potential for tissue regeneration and are capable of giving rise to osteoblast-/cementoblast-like cells, adipocytes, chondrogenic cells, neurogenic lineage cells, endothelial cells, cardiac myocytes, and Schwann cells in vitro [62]. For stem cell identity and fate commitment, Roguljic and colleagues identified $\alpha$ SMA as a marker of PDLSCs in vivo [82]. $\alpha \mathrm{SMA}^{+}$PDLSCs expanded over time and mainly gave rise to cells in the apical region. Following periodontal ligament injury, PDLSCs proliferated and generated mature cementoblasts, osteoblasts, and fibroblasts within the periodontium. However, $\alpha \mathrm{SMA}^{+}$PDLSCs only made minor contributions to periodontium homeostasis and repair. Yuan and colleagues used Axin2 to track progenitor cells in the periodontal ligament and reported that PDLSCs are responsive to Wnt signaling [83]. Axin $2^{+}$PDLSCs remain quiescent under physiological conditions and differentiate into osteoblastic cells for alveolar bone repair when tooth extraction injury occurs. Most recently, using genetic fate mapping, Men et al. identified Gli1 ${ }^{+}$ PDLSCs in adult mouse molars that gave rise to periodontal ligament, alveolar bone, and cementum in both the homeostatic state and during injury repair [84]. Gli1+ PDLSCs are enriched in the apical tooth region and surround the neurovascular bundle, and they are activated by canonical Wnt signaling. Sclerostin secreted by alveolar bone osteocytes inhibits Wnt signaling. Occlusal force can inhibit sclerostin secretion. Therefore, a feedback loop that regulates stem cell activities is present in the stem cell niche, where occlusal force-mediated inhibition of sclerostin secretion by alveolar bone osteocytes promotes $\mathrm{Glil}^{+}$cell maintenance and activation. The authors also compared other stem cell markers with Gli1. They concluded that labeling of Glil more efficiently identified PDLSCs compared to labeling of $\alpha \mathrm{SMA}$, LepR, NG2, and Pdgfr $\alpha$. Using an inducible Cre system and immunostaining, they also reported that LepR ${ }^{+}, \mathrm{NG}_{2}^{+}$, and Pdgfr $\alpha^{+}$cells are descendants of Gli1+ cells in the periodontal ligament.

\section{Application of Craniofacial Stem Cells}

Stem cells for tissue regeneration have been widely exploited and are mainly applied in three ways: direct transplantation of a specific type of stem cells, combined application of different types of stem cells, and the use of stem cells in combination with biological scaffolds. However, the regenerative techniques used in the long bone cannot be easily extended to craniofacial applications because the microenvironment of craniofacial tissue is quite different from that of the long bone. Oral pathological factors (e.g., microorganisms, nicotine, and bisphosphonate) greatly affect the biological behavior of craniofacial stem cells. For instance, Kim et al. found that excessive nicotine intake will induce the vacuolation of jawbone MSCs and impair their proliferation and differentiation capacity [85]. Akintoye et al. found that jawbone MSC self-renewal and proliferation ability were impaired when the MSCs were treated with bisphosphonate, which might be associated with the pathogenesis of bisphosphonate-associated osteonecrosis. Therefore, to minimize potential damage, the use of appropriate ways to amplify and induce stem cells to differentiate toward the osteoblast lineage is particularly important. Wang et al. reported that vitamin $\mathrm{C}$ and vitamin $\mathrm{D}$ were ideal stimu- lants of craniofacial PDLSCs for osteoblast differentiation in vitro [26]. Naung et al. proposed a protocol to cultivate palate periosteum-derived MSCs in serum-free and xenofree medium, which could become a useful source of MSCs for clinical applications [86]. Moreover, a recent study found that induced pluripotent stem cells (iPSCs) generated from human jaw periosteum cells expressed MSC markers and possessed strong mineralization ability [87].

The application of MSCs for regenerative medicine should be performed with caution because the stem cells obtained and expanded with plastic culture are highly heterogeneous and might not be intrinsically multipotent [88]. It should be noted that the minimal criteria, including adherence to the plastic plate, induction of multidirectional differentiation, and detection of appropriate surface marker profiles, are not sufficient to identify bona fide stem cells [89]. Some quiescent stem cells might not readily adhere to the plastic, and the heterogeneous cell mixture contains lineage-committed stem cells that give rise to cells of native tissue origin. For instance, bone marrow stem cells show an intrinsic propensity for differentiation toward osteolineages [90]. The surface markers of bone marrow stem cells are specific for fibroblast-like cells rather than stem cells [88]. Therefore, in vivo clonal assays and fate mapping are essential to identify bona fide stem cells [38]. Future studies are warranted to identify the stem cell properties of craniofacial stem cells, which will benefit clinical applications.

\section{Conclusions}

This review describes the stem cells found in the craniofacial periosteum, craniofacial bone marrow, TMJ, cranial suture, and periodontal ligament. Craniofacial stem cells express specific cell surface determinants, possess a low self-renewal rate, and show multidifferentiation ability. They rapidly proliferated and differentiated into osteoblasts in response to injury. In addition, craniofacial tissues are easily obtained from human jawbones with minimal invasiveness when clinicians perform implant surgeries, tooth extractions, and periodontal surgeries. All these results indicated that craniofacial stem cells are an ideal resource for tissue engineering.

However, the current research on craniofacial stem cells is still inadequate and lacks depth. Specific cell markers for the isolation of stem cells are still lacking. In vivo clonal assays and lineage fate mapping are warranted in future studies, which could facilitate the therapeutic application of craniofacial stem cells in vivo and in the clinic.

\section{Conflicts of Interest}

The authors declare that they have no competing interests.

\section{Authors' Contributions}

Geru Zhang and Qiwen Li contributed equally to this work.

\section{Acknowledgments}

This work was supported by grants from the National Natural Science Foundation of China (Grant Number: NSFC 
81901042) and China Postdoctoral Science Foundation (Grant Number: 2019M653443) to Shiwen Zhang.

\section{References}

[1] K. S. Boehm, M. al-Taha, A. Morzycki, O. A. Samargandi, S. alYouha, and M. R. LeBlanc, "Donor site morbidities of iliac crest bone graft in craniofacial surgery: a systematic review," Annals of Plastic Surgery, vol. 83, no. 3, pp. 352-358, 2019.

[2] W. Zhang and P. C. Yelick, "Craniofacial tissue engineering," Cold Spring Harbor Perspectives in Medicine, vol. 8, no. 1, 2018.

[3] J. A. Fearon, D. Griner, K. Ditthakasem, and M. Herbert, "Autogenous bone reconstruction of large secondary skull defects," Plastic and Reconstructive Surgery, vol. 139, no. 2, pp. 427-438, 2017.

[4] J. C. Melville, V. A. Mañón, C. Blackburn, and S. Young, "Current methods of maxillofacial tissue engineering," Oral and Maxillofacial Surgery Clinics of North America, vol. 31, no. 4, pp. 579-591, 2019.

[5] Y. Fillingham and J. Jacobs, "Bone grafts and their substitutes," The Bone \& Joint Journal, vol. 98-B, 1_Supple_A, pp. 6-9, 2016.

[6] R. Dimitriou, E. Jones, D. McGonagle, and P. V. Giannoudis, "Bone regeneration: current concepts and future directions," BMC Medicine, vol. 9, no. 1, 2011.

[7] A. Andrzejewska, B. Lukomska, and M. Janowski, "Concise review: mesenchymal stem cells: from roots to boost," Stem Cells, vol. 37, no. 7, pp. 855-864, 2019.

[8] X. Fu, G. Liu, A. Halim, Y. Ju, Q. Luo, and G. Song, "Mesenchymal stem cell migration and tissue repair," Cell, vol. 8, no. 8, p. 784, 2019.

[9] J. Galipeau and L. Sensébé, "Mesenchymal stromal cells: clinical challenges and therapeutic opportunities," Cell Stem Cell, vol. 22, no. 6, pp. 824-833, 2018.

[10] R. Yang, T. Yu, and Y. Zhou, "Interplay between craniofacial stem cells and immune stimulus," Stem Cell Research \& Therapy, vol. 8, no. 1, p. 147, 2017.

[11] J. L. Spees, R. H. Lee, and C. A. Gregory, "Mechanisms of mesenchymal stem/stromal cell function," Stem Cell Research \& Therapy, vol. 7, no. 1, p. 125, 2016.

[12] C. Harrell, C. Fellabaum, N. Jovicic, V. Djonov, N. Arsenijevic, and V. Volarevic, "Molecular mechanisms responsible for therapeutic potential of mesenchymal stem cell-derived secretome," Cell, vol. 8, no. 5, p. 467, 2019.

[13] M. De Luca, A. Aiuti, G. Cossu, M. Parmar, G. Pellegrini, and P. G. Robey, "Advances in stem cell research and therapeutic development," Nature Cell Biology, vol. 21, no. 7, pp. 801$811,2019$.

[14] A. Ho-Shui-Ling, J. Bolander, L. E. Rustom, A. W. Johnson, F. P. Luyten, and C. Picart, "Bone regeneration strategies: engineered scaffolds, bioactive molecules and stem cells current stage and future perspectives," Biomaterials, vol. 180, pp. 143-162, 2018.

[15] M. Dominici, K. le Blanc, I. Mueller et al., "Minimal criteria for defining multipotent mesenchymal stromal cells. The International Society for Cellular Therapy position statement," Cytotherapy, vol. 8, no. 4, pp. 315-317, 2006.

[16] M. Kassem and P. Bianco, "Skeletal stem cells in space and time," Cell, vol. 160, no. 1-2, pp. 17-19, 2015.

[17] F. Kawecki, W. P. Clafshenkel, M. Fortin, F. A. Auger, and J. Fradette, "Biomimetic tissue-engineered bone substitutes for maxillofacial and craniofacial repair: the potential of cell sheet technologies," Advanced Healthcare Materials, vol. 7, no. 6, article e1700919, 2018.

[18] P. T. Sharpe, "Dental mesenchymal stem cells," Development, vol. 143, no. 13, pp. 2273-2280, 2016.

[19] J. Liu, F. Yu, Y. Sun et al., "Concise reviews: characteristics and potential applications of human dental tissue-derived mesenchymal stem cells," Stem Cells, vol. 33, no. 3, pp. 627-638, 2015.

[20] H. Zhao and Y. Chai, "Stem cells in teeth and craniofacial bones," Journal of Dental Research, vol. 94, no. 11, pp. 14951501, 2015.

[21] D. Wang, J. R. Gilbert, X. Zhang, B. Zhao, D. F. E. Ker, and G. M. Cooper, "Calvarial versus long bone: implications for tailoring skeletal tissue engineering," Tissue Engineering Part B: Reviews, vol. 26, no. 1, pp. 46-63, 2020.

[22] B. Hernández-Monjaraz, E. Santiago-Osorio, A. Monroy-García, E. Ledesma-Martínez, and V. Mendoza-Núñez, "Mesenchymal stem cells of dental origin for inducing tissue regeneration in periodontitis: a mini-review," International Journal of Molecular Sciences, vol. 19, no. 4, p. 944, 2018.

[23] M. Yang, H. Zhang, and R. Gangolli, “Advances of mesenchymal stem cells derived from bone marrow and dental tissue in craniofacial tissue engineering," Current Stem Cell Research \& Therapy, vol. 9, no. 3, pp. 150-161, 2014.

[24] D. L. Alge, D. Zhou, L. L. Adams et al., "Donor-matched comparison of dental pulp stem cells and bone marrow-derived mesenchymal stem cells in a rat model," Journal of Tissue Engineering and Regenerative Medicine, vol. 4, no. 1, pp. 73-81, 2010.

[25] R. C. Ransom, A. C. Carter, A. Salhotra et al., "Mechanoresponsive stem cells acquire neural crest fate in jaw regeneration," Nature, vol. 563, no. 7732, pp. 514-521, 2018.

[26] Y.-L. Wang, A. Hong, T.-H. Yen, and H.-H. Hong, "Isolation of mesenchymal stem cells from human alveolar periosteum and effects of vitamin D on osteogenic activity of periosteum-derived cells," Journal of Visualized Experiments, vol. 135, article e57166, 2018.

[27] O. D. de Lageneste, A. Julien, R. Abou-Khalil et al., "Periosteum contains skeletal stem cells with high bone regenerative potential controlled by periostin," Nature Communications, vol. 9, no. 1, 2018.

[28] Z. Lin, A. Fateh, D. M. Salem, and G. Intini, "Periosteum," Journal of Dental Research, vol. 93, no. 2, pp. 109-116, 2013.

[29] S. Mouraret, E. von Kaeppler, C. Bardet et al., "The potential for vertical bone regeneration via maxillary periosteal elevation," Journal of Clinical Periodontology, vol. 41, no. 12, pp. 1170-1177, 2014.

[30] S. Debnath, A. R. Yallowitz, J. McCormick et al., "Discovery of a periosteal stem cell mediating intramembranous bone formation," Nature, vol. 562, no. 7725, pp. 133-139, 2018.

[31] L. C. Ortinau, H. Wang, K. Lei et al., "Identification of functionally distinct $\mathrm{Mx} 1+\alpha \mathrm{SMA}+$ periosteal skeletal stem cells," Cell Stem Cell, vol. 25, no. 6, pp. 784-796.e5, 2019.

[32] S. O. Akintoye, T. Lam, S. Shi, J. Brahim, M. T. Collins, and P. G. Robey, "Skeletal site-specific characterization of orofacial and iliac crest human bone marrow stromal cells in same individuals," Bone, vol. 38, no. 6, pp. 758-768, 2006.

[33] Y. Du, F. Jiang, Y. Liang et al., "The angiogenic variation of skeletal site-specific human BMSCs from same alveolar cleft 
patients: a comparative study," Journal of Molecular Histology, vol. 47, no. 2, pp. 153-168, 2016.

[34] M. Kanawa, A. Igarashi, K. Fujimoto et al., "Genetic markers can predict chondrogenic differentiation potential in bone marrow-derived mesenchymal stromal cells," Stem Cells International, vol. 2018, Article ID 9530932, 9 pages, 2018.

[35] T. Matsubara, K. Suardita, M. Ishii et al., "Alveolar bone marrow as a cell source for regenerative medicine: differences between alveolar and iliac bone marrow stromal cells," Journal of Bone and Mineral Research, vol. 20, no. 3, pp. 399-409, 2005.

[36] B. O. Zhou, R. Yue, M. M. Murphy, J. G. Peyer, and S. J. Morrison, "Leptin-receptor-expressing mesenchymal stromal cells represent the main source of bone formed by adult bone marrow," Cell Stem Cell, vol. 15, no. 2, pp. 154-168, 2014.

[37] D. L. Worthley, M. Churchill, J. T. Compton et al., "Gremlin 1 identifies a skeletal stem cell with bone, cartilage, and reticular stromal potential," Cell, vol. 160, no. 1-2, pp. 269-284, 2015.

[38] C. K. F. Chan, E. Y. Seo, J. Y. Chen et al., "Identification and specification of the mouse skeletal stem cell," Cell, vol. 160, no. 1-2, pp. 285-298, 2015.

[39] Y. Shi, G. He, W. C. Lee, J. A. McKenzie, M. J. Silva, and F. Long, "Gli1 identifies osteogenic progenitors for bone formation and fracture repair," Nature Communications, vol. 8, no. 1, p. 2043, 2017.

[40] D. Zhang, S. Zhang, J. Wang et al., "LepR-expressing stem cells are essential for alveolar bone regeneration," Journal of Dental Research, no. article 002203452093283, 2020.

[41] Z. Liu, X. Long, J. Li, L. Wei, Z. Gong, and W. Fang, "Differentiation of temporomandibular joint synovial mesenchymal stem cells into neuronal cells in vitro: an in vitro study," Cell Biology International, vol. 35, no. 1, pp. 87-91, 2011.

[42] N. Koyama, Y. Okubo, K. Nakao, K. Osawa, K. Fujimura, and K. Bessho, "Pluripotency of mesenchymal cells derived from synovial fluid in patients with temporomandibular joint disorder," Life Sciences, vol. 89, no. 19-20, pp. 741-747, 2011.

[43] E. Xiao, J. M. Li, Y. B. Yan et al., "Decreased osteogenesis in stromal cells from radiolucent zone of human TMJ ankylosis," Journal of Dental Research, vol. 92, no. 5, pp. 450-455, 2013.

[44] Y. P. Sun, Y. H. Zheng, W. J. Liu, Y. L. Zheng, and Z. G. Zhang, "Synovium fragment-derived cells exhibit characteristics similar to those of dissociated multipotent cells in synovial fluid of the temporomandibular joint," PLoS One, vol. 9, no. 7, article e101896, 2014.

[45] M. C. Embree, M. Chen, S. Pylawka et al., "Exploiting endogenous fibrocartilage stem cells to regenerate cartilage and repair joint injury," Nature Communications, vol. 7, no. 1, 2016.

[46] T. Maruyama, J. Jeong, T. J. Sheu, and W. Hsu, "Stem cells of the suture mesenchyme in craniofacial bone development, repair and regeneration," Nature Communications, vol. 7, no. $1,2016$.

[47] E. Durham, R. N. Howie, N. Larson, A. LaRue, and J. Cray, "Pharmacological exposures may precipitate craniosynostosis through targeted stem cell depletion," Stem Cell Research, vol. 40, p. 101528, 2019.

[48] E. L. Durham, R. N. Howie, R. Houck et al., "Involvement of calvarial stem cells in healing: a regional analysis of large cranial defects," Wound Repair and Regeneration, vol. 26, no. 5, pp. 359-365, 2018.

[49] D. H. Doro, A. E. Grigoriadis, and K. J. Liu, "Calvarial suturederived stem cells and their contribution to cranial bone repair," Frontiers in Physiology, vol. 8, p. 956, 2017.
[50] S. Park, H. Zhao, M. Urata, and Y. Chai, "Sutures possess strong regenerative capacity for calvarial bone injury," Stem Cells and Development, vol. 25, no. 23, pp. 1801-1807, 2016.

[51] H. Zhao, J. Feng, T. V. Ho, W. Grimes, M. Urata, and Y. Chai, "The suture provides a niche for mesenchymal stem cells of craniofacial bones," Nature Cell Biology, vol. 17, no. 4, pp. 386-396, 2015.

[52] K. Wilk, S. C. A. Yeh, L. J. Mortensen et al., "Postnatal calvarial skeletal stem cells expressing PRX1 reside exclusively in the calvarial sutures and are required for bone regeneration," Stem Cell Reports, vol. 8, no. 4, pp. 933-946, 2017.

[53] S. O. Akintoye, "The distinctive jaw and alveolar bone regeneration," Oral Diseases, vol. 24, no. 1-2, pp. 49-51, 2018.

[54] S. Gronthos, M. Mankani, J. Brahim, P. G. Robey, and S. Shi, "Postnatal human dental pulp stem cells (DPSCs) in vitro and in vivo," Proceedings of the National Academy of Sciences of the United States of America, vol. 97, no. 25, pp. 1362513630, 2000.

[55] M. Miura, S. Gronthos, M. Zhao et al., "SHED: stem cells from human exfoliated deciduous teeth," Proceedings of the National Academy of Sciences of the United States of America, vol. 100, no. 10, pp. 5807-5812, 2003.

[56] B.-M. Seo, M. Miura, S. Gronthos et al., "Investigation of multipotent postnatal stem cells from human periodontal ligament," Lancet, vol. 364, no. 9429, pp. 149-155, 2004.

[57] C. Morsczeck, W. Götz, J. Schierholz et al., "Isolation of precursor cells (PCs) from human dental follicle of wisdom teeth," Matrix Biology, vol. 24, no. 2, pp. 155-165, 2005.

[58] W. Sonoyama, Y. Liu, T. Yamaza et al., "Characterization of the apical papilla and its residing stem cells from human immature permanent teeth: a pilot study," Journal of Endodontia, vol. 34, no. 2, pp. 166-171, 2008.

[59] E. Ikeda, K. Yagi, M. Kojima et al., "Multipotent cells from the human third molar: feasibility of cell-based therapy for liver disease," Differentiation, vol. 76, no. 5, pp. 495-505, 2008.

[60] Q. Z. Zhang, W. R. Su, S. H. Shi et al., "Human gingiva-derived mesenchymal stem cells elicit polarization of $\mathrm{m} 2$ macrophages and enhance cutaneous wound healing," Stem Cells, vol. 28, no. 10, pp. 1856-1868, 2010.

[61] E. Karaöz, B. N. Doğan, A. Aksoy et al., "Isolation and in vitro characterisation of dental pulp stem cells from natal teeth," Histochemistry and Cell Biology, vol. 133, no. 1, pp. 95-112, 2010.

[62] S. Aydin and F. Sahin, "Stem cells derived from dental tissues," Advances in Experimental Medicine and Biology, vol. 1144, pp. 123-132, 2019.

[63] F. M. Chen, L. N. Gao, B. M. Tian et al., "Treatment of periodontal intrabony defects using autologous periodontal ligament stem cells: a randomized clinical trial," Stem Cell Research \& Therapy, vol. 7, no. 1, 2016.

[64] L. Winning, I. A. El Karim, and F. T. Lundy, "A comparative analysis of the osteogenic potential of dental mesenchymal stem cells," Stem Cells and Development, vol. 28, no. 15, pp. 1050-1058, 2019.

[65] M. Khoshhal, I. Amiri, and L. Gholami, "Comparison of in vitro properties of periodontal ligament stem cells derived from permanent and deciduous teeth," Journal of Dental Research, Dental Clinics, Dental Prospects, vol. 11, no. 3, pp. 140-148, 2017.

[66] J. S. Song, S. O. Kim, S. H. Kim et al., "In vitro and in vivo characteristics of stem cells derived from the periodontal ligament 
of human deciduous and permanent teeth," Tissue Engineering. Part A, vol. 18, no. 19-20, pp. 2040-2051, 2012.

[67] N. Liu, S. Shi, M. Deng et al., "High levels of $\beta$-catenin signaling reduce osteogenic differentiation of stem cells in inflammatory microenvironments through inhibition of the noncanonical Wnt pathway," Journal of Bone and Mineral Research: the official journal of the American Society for Bone and Mineral Research, vol. 26, no. 9, pp. 2082-2095, 2011.

[68] B. Li, Y. Zhang, Q. Wang et al., "Periodontal ligament stem cells modulate root resorption of human primary teeth via Runx2 regulating RANKL/OPG system," Stem Cells and Development, vol. 23, no. 20, pp. 2524-2534, 2014.

[69] H. Kato, Y. Taguchi, K. Tominaga, M. Umeda, and A. Tanaka, "Porphyromonas gingivalis LPS inhibits osteoblastic differentiation and promotes pro-inflammatory cytokine production in human periodontal ligament stem cells," Archives of Oral Biology, vol. 59, no. 2, pp. 167-175, 2014.

[70] Q. Jia, W. Jiang, and L. Ni, “Down-regulated non-coding RNA (lncRNA-ANCR) promotes osteogenic differentiation of periodontal ligament stem cells," Archives of Oral Biology, vol. 60, no. 2, pp. 234-241, 2015.

[71] L. Wang, F. Wu, Y. Song et al., "Long noncoding RNA related to periodontitis interacts with miR-182 to upregulate osteogenic differentiation in periodontal mesenchymal stem cells of periodontitis patients," Cell Death \& Disease, vol. 7, no. 8, article e2327, 2016.

[72] F. Cao, J. Zhan, X. Chen, K. Zhang, R. Lai, and Z. Feng, "miR214 promotes periodontal ligament stem cell osteoblastic differentiation by modulating Wnt/ $\beta$-catenin signaling," Molecular Medicine Reports, vol. 16, no. 6, pp. 9301-9308, 2017.

[73] X. Gu, M. Li, Y. Jin, D. Liu, and F. Wei, "Identification and integrated analysis of differentially expressed lncRNAs and circRNAs reveal the potential ceRNA networks during PDLSC osteogenic differentiation," BMC Genetics, vol. 18, no. 1, p. 100, 2017.

[74] Y. He, C. X. Jian, H. Y. Zhang et al., "Hypoxia enhances periodontal ligament stem cell proliferation via the MAPK signaling pathway," Genetics and Molecular Research, vol. 15, no. 4, 2016.

[75] W. Yan, Y. Cao, H. Yang et al., "CB1 enhanced the osteo/dentinogenic differentiation ability of periodontal ligament stem cells via p38 MAPK and JNK in an inflammatory environment," Cell Proliferation, vol. 52, no. 6, article e12691, 2019.

[76] X. Y. Xu, X. T. He, J. Wang et al., "Role of the P2X7 receptor in inflammation-mediated changes in the osteogenesis of periodontal ligament stem cells," Cell Death \& Disease, vol. 10, no. 1, p. 20, 2019.

[77] Y. Xu, C. Ren, X. Zhao, W. Wang, and N. Zhang, "microRNA132 inhibits osteogenic differentiation of periodontal ligament stem cells via GDF5 and the NF- $\kappa$ B signaling pathway," Pathology - Research and Practice, vol. 215, no. 12, p. 152722, 2019.

[78] R. Zhang, Q. Liang, W. Kang, and S. Ge, "Metformin facilitates the proliferation, migration, and osteogenic differentiation of periodontal ligament stem cells in vitro," Cell Biology International, vol. 44, no. 1, pp. 70-79, 2019.

[79] Z. Chen and H. L. Liu, "Restoration of miR-1305 relieves the inhibitory effect of nicotine on periodontal ligament-derived stem cell proliferation, migration, and osteogenic differentiation," Journal of Oral Pathology \& Medicine, vol. 46, no. 4, pp. 313-320, 2017.
[80] T. K. Ng, L. Huang, D. Cao et al., "Cigarette smoking hinders human periodontal ligament-derived stem cell proliferation, migration and differentiation potentials," Scientific Reports, vol. 5, no. 1, p. 7828, 2015.

[81] L. L. Ramenzoni, G. Russo, M. D. Moccia, T. Attin, and P. R. Schmidlin, "Periodontal bacterial supernatants modify differentiation, migration and inflammatory cytokine expression in human periodontal ligament stem cells," PLoS One, vol. 14, no. 7, article e0219181, 2019.

[82] H. Roguljic, B. G. Matthews, W. Yang, H. Cvija, M. Mina, and I. Kalajzic, "In vivo identification of periodontal progenitor cells," Journal of Dental Research, vol. 92, no. 8, pp. 709-715, 2013.

[83] X. Yuan, X. Pei, Y. Zhao, U. S. Tulu, B. Liu, and J. A. Helms, “A Wnt-responsive PDL population effectuates extraction socket healing," Journal of Dental Research, vol. 97, no. 7, pp. 803809, 2018.

[84] Y. Men, Y. Wang, Y. Yi et al., "Gli1+ periodontium stem cells are regulated by osteocytes and occlusal force," Developmental Cell, 2020.

[85] B. S. Kim, S. J. Kim, H. J. Kim et al., "Effects of nicotine on proliferation and osteoblast differentiation in human alveolar bone marrow-derived mesenchymal stem cells," Life Sciences, vol. 90, no. 3-4, pp. 109-115, 2012.

[86] N. Y. Naung, W. Duncan, R. D. Silva, and D. Coates, "Localization and characterization of human palatal periosteum stem cells in serum-free, xeno-free medium for clinical use," European Journal of Oral Sciences, vol. 127, no. 2, pp. 99-111, 2019.

[87] F. Umrath, H. Steinle, M. Weber et al., "Generation of iPSCs from jaw periosteal cells using self-replicating RNA," International Journal of Molecular Sciences, vol. 20, no. 7, p. 1648, 2019.

[88] T. H. Ambrosi, M. T. Longaker, and C. K. F. Chan, "A revised perspective of skeletal stem cell biology," Frontiers in Cell and Development Biology, vol. 7, p. 189, 2019.

[89] P. Bianco and P. G. Robey, "Skeletal stem cells," Development, vol. 142, no. 6, pp. 1023-1027, 2015.

[90] A. Rauch, A. K. Haakonsson, J. G. S. Madsen et al., "Osteogenesis depends on commissioning of a network of stem cell transcription factors that act as repressors of adipogenesis," Nature Genetics, vol. 51, no. 4, pp. 716-727, 2019. 